\title{
Antithrombotic management in patients with percutaneous coronary intervention requiring oral anticoagulation
}

\author{
Jarosław Zalewski ${ }^{1}$, Anetta Undas ${ }^{2}$ \\ ${ }^{1}$ Department of Coronary Disease, John Paul II Hospital, Jagiellonian University Medical College, Krakow, Poland \\ ${ }^{2}$ Institute of Cardiology, Jagiellonian University Medical College and Centre for Research and Medical Technology, John Paul II Hospital, \\ Krakow, Poland
}

\begin{abstract}
A bstract
The dynamic evolution of therapeutic options including the use of vitamin $\mathrm{K}$ antagonists (VKA), non-vitamin $\mathrm{K}$ oral anticoagulants (NOAC), more potent antiplatelet drugs as well as new generation drug-eluting stents could lead to the view that the current recommendations on the management of patients with percutaneous coronary intervention $(\mathrm{PCI})$ requiring oral anticoagulation do not keep up with the results of several clinical studies published within the last 5 years. In the present overview, we summarize the recent advances in antithrombotic management used in atrial fibrillation patients undergoing PCI for stable coronary artery disease or acute coronary syndrome (ACS). The safety and efficacy of prasugrel and ticagrelor taken with oral anticoagulants also remain to be established in randomized trials; therefore the $\mathrm{P}_{2} \mathrm{Y}_{12}$ inhibitor clopidogrel on top of aspirin or without is now recommended to be used together with a VKA or NOAC. It is still unclear which dose of a NOAC in combination with antiplatelet agents and different stents should be used in this clinical setting and whether indeed NOAC are safer compared with VKA in such cardiovascular patients. Moreover, we discuss the use of anticoagulation in addition to antiplatelet therapy for secondary prevention in patients with ACS. To minimize bleeding risk in anticoagulated patients following PCI or ACS, the right agent should be prescribed to the right patient at the right dose and supported by regular clinical evaluation and laboratory testing, especially assessment of renal function when a NOAC is used.
\end{abstract}

Key words: percutaneous coronary intervention, antiplatelet therapy, atrial fibrillation, non-vitamin K oral anticoagulants, vitamin $\mathrm{K}$ antagonist.

\section{Introduction}

The coexistence of coronary artery disease (CAD) requiring revascularization and thromboembolic disorders requiring oral anticoagulation (OAC) is a common and complex clinical issue. The most frequent indication for $\mathrm{OAC}$ is atrial fibrillation (AF) [1]. The incidence of percutaneous coronary intervention $(\mathrm{PCI})$ or coronary artery bypass surgery (CABG) in patients with $A F$ is about $20 \%$ [2]; thus in Europe 1-2 million AF patients who are on OAC may require revascularization. Based on the results of a meta-analysis involving 120,566 patients with ST-segment elevation myocardial infarction (STEMI) or non-STsegment elevation myocardial infarction (NSTEMI) [3], AF was present in $7.5 \%$ of the overall study population and was associated with a higher 7-day mortality rate of $5.1 \%$ compared with $1.6 \%$ for those without AF. In multivariable analysis AF was also associated with 2.4 times for STEMI and 1.7 times for NSTEMI higher risk of long-term mortality between day 8 and 1 year. According to the results of subanalysis of the HORIZONS-AMI (Harmonizing Outcomes with Revascularization and Stents in Acute Myocardial Infarction) trial comparing different modern antithrombotic regimens and stents [4], patients with new-onset AF during STEMI had higher 3 -year rates of mortality $(11.9 \%$ vs. $6.3 \%, p=0.01)$, reinfarction ( $16.4 \%$ vs. $7.0 \%, p<0.0001)$, stroke (5.8\% vs. $1.5 \%, p<0.0001)$, and major bleeding (20.9\% vs. $8.2 \%$, $p<0.0001)$. Moreover, recently the combination of reduced doses of non-vitamin $\mathrm{K}$ oral anticoagulants (NOAC), or direct oral anticoagulants (DOAC), with dual

\section{Corresponding author:}

Jarosław Zalewski MD, PhD, Department of Coronary Disease, John Paul II Hospital, Jagiellonian University Medical College, 80 Pradnicka St, 31-202 Krakow, Poland, phone: +48 602475 182, e-mail: jzalews@szpitaljp2.krakow.pl

Received: 8.10.2016, accepted: 31.10.2016. 
antiplatelet therapy (DAPT), has been tested in patients after acute coronary syndrome (ACS).

The dynamic evolution of therapeutic options including the use of vitamin $\mathrm{K}$ antagonists (VKA), NOAC, more potent antiplatelet drugs as well as new generation drug-eluting stents (DES) could lead to the view that the current recommendations on the management in this group of patients do not keep up with the recent advances of modern pharmaco-invasive medicine [1, 5-7]. In the present overview, we summarize the current status of antithrombotic management in AF patients undergoing $\mathrm{PCI}$ for stable CAD or ACS.

\section{Peri-procedural antithrombotic management}

Periprocedural management both in stable CAD and ACS patients on OAC remains a challenge. It requires the balance of the risk of thromboembolic complications, intracoronary thrombosis associated with anticoagulation interruption and the risk of periprocedural bleeding related to the bridging anticoagulation and antiplatelet therapy [8-10]. Regardless of the type of chronic anticoagulation therapy, in patients on $\mathrm{OAC}$, during $\mathrm{PCl}$ for both ACS and stable CAD, i) radial access should be the default to minimize the risk of access-related bleeding, ii) new generation DES or bare metal stents (BMS) are recommended if triple therapy is planned, iii) routine use of ticagrelor or prasugrel is discouraged because of their unknown safety profile in association with VKA or NOAC, and iv) GP IIb/IIla inhibitors should be avoided unless for bail-out situations.

For many years, bridging therapy with parenteral heparins instead of the uninterrupted treatment with VKA has been applied during $\mathrm{PCl}$. The bridging strategy rationale was based on the replacement of oral warfarin by parenteral agents of short half-life, of rapid onset of their action during the procedure, and a relative easy strategy to reverse anticoagulant effects. However, for this empirical approach there is a lack of robust evidence based on randomized, controlled studies.

There is no doubt that in patients on OAC undergoing $\mathrm{PCl}$, the risk stratification of thrombosis and bleeding needs to be weighed first. However, existing scores such as $\mathrm{CHA}_{2} \mathrm{DS}_{2}$-VASC and HAS-BLED have not been validated in case of anticoagulation interruption or potential bridging therapy. In the prospective multicenter AFCAS (Atrial Fibrillation undergoing Coronary Artery Stenting) registry, bleeding complications and major adverse cardiac and cerebrovascular events (MACCE) including death, myocardial infarction (MI), target vessel revascularization, stent thrombosis and stroke have been assessed during a 30-day follow-up period in 290 patients treated with uninterrupted OAC with VKA and in 161 patients with bridging therapy (BT) conducted for a median of 3 days before coronary artery stenting [11]. The international normalized ratio (INR) before PCI was higher (2.3 vs. 1.8, $p<0.001)$ and radial access was more common (43\% vs. $14 \%, p<0.001)$ in the uninterrupted OAC group. In the BT periprocedural unfractionated or low-molecular weight heparin (LMWH) bolus (70\% vs. 48\%, $p<0.001)$ and glycoprotein IIb/IIla inhibitors ( $26 \%$ vs. $7 \%, p<0.001$ ) were given more often as compared with the uninterrupted OAC group. Aspirin was started not later than during PCI in almost all patients, and less than $2 \%$ of patients in both groups did not receive periprocedural clopidogrel. Finally, at discharge triple therapy of aspirin, clopidogrel plus OAC and/or LMWH was the most frequent regimen after $\mathrm{PCl}$ in both $\mathrm{BT}$ and uninterrupted OAC groups $(76 \%$ vs. $90 \%, p<0.001)$. On the other hand, dual therapy with clopidogrel plus OAC and/or LMWH was used in $6 \%$ of uninterrupted OAC and $12 \%$ of BT $(p<0.001)$. The length of hospitalization after $\mathrm{PCl}$ was longer in the $\mathrm{BT}$ group both in elective and in acute patients (3.2 vs. 1.9 days and 7.0 vs. 5.3 days, respectively; $p<0.05$ for both). Overall bleeding complications tended to be more common in the BT group ( $19 \%$ vs. $12 \%, p=0.07$ ), with no significant difference in the rate of major bleeding (2.5\% vs. $1.4 \%$ ) or MACCE (6.2\% vs. 3.8\%). However, after propensity adjustment, the frequency of bleeding complications or MACCE was similar. Multivariate analysis showed that femoral access, ACS and history of bleeding were independent predictors of bleeding complications and that ACS was the only independent predictor for MACCE. Periprocedural INR was not associated with bleeding or MACCE. The results of the AFCAS registry suggest that uninterrupted OAC does not increase periprocedural complications during coronary stenting and is a simple and cost-effective alternative to conventional heparin bridging.

Similar findings were obtained in the subanalysis of the WOEST study [12]. The frequency of bleeding complications and MACCE were assessed in 241 patients treated with uninterrupted OAC and in 322 patients with BT. Radial access was more common in the uninterrupted OAC group. Moreover, the periprocedural use of LMWH (30\% vs. $16 \%, p<0.001)$ and GP IIb/IIla inhibitors (14\% vs. $3 \%, p<0.001$ ) was significantly lower in the uninterrupted OAC group, whereas the unfractionated heparin (UFH) bolus was given more frequently (90\% vs. 94\%, $p<0.001)$. The periprocedural INR was higher in the uninterrupted OAC group (2.5 vs. $1.5, p<0.001)$. The length of hospitalization after $\mathrm{PCl}$ was the same for both groups after elective $\mathrm{PCI}$ (median 1 day), but was longer in ACS patients in the uninterrupted OAC group (medians 3 vs. 2 days). After 30 days, as well as after one year, there were no differences in bleeding events $(p=0.51, p=$ 0.12 , respectively). After 1 year, MACCE tended to be less frequent in the uninterrupted OAC group (12\% vs. $16 \%$, $p=0.16$ ). Additionally, adjustment with a propensity score revealed no significant differences. Periprocedural INR was not associated with bleeding or MACCE. Also the WOEST substudy indicates that in patients treated with 
long-term $\mathrm{OAC}$ who undergo $\mathrm{PCl}$, periprocedural continuation of OAC is safe and effective.

Importantly, despite a significantly higher value of periprocedural INR, uninterrupted OAC with VKA did not increase the rate of bleeding complications at all. Moreover, in the AFCAS substudy BT was associated with a higher rate of access-site bleeding, whereas in both AFCAS and WOEST substudies uninterrupted OAC was associated with similar rates of stent thrombosis. An advantage of uninterrupted VKA therapy during $\mathrm{PCl}$ might be avoidance of potential thromboembolic complications associated with a period of subtherapeutic anticoagulation and elimination of a period of a potential transient prothrombotic state due to protein $C$ and $S$ suppression after warfarin re-initiation. Moreover, uninterrupted OAC with VKA may offer an economic benefit related to shorter hospitalization. Based on the above-presented results in patients with therapeutic INR 2-3.0 during PCI dual antiplatelet therapy, no additional heparin bolus and radial access seem the first choice strategy [10, 13]. An additional UFH bolus or bivalirudin (especially at high risk of bleeding) may be considered in patients with INR $<2.0$, complex lesion $\mathrm{PCl}$ or prolonged procedure; however, glycoprotein IIb/IIla inhibitors should be avoided (Figure 1).

The experience with performing $\mathrm{PCl}$ in patients treated with NOAC is limited. In a small, phase Ila, randomized study, 50 stable patients on standard DAPT were randomized $(2: 2: 1)$ to either pre-procedural dabigatran
$110 \mathrm{mg}$ BID or $150 \mathrm{mg}$ BID or standard intraprocedural UFH [14]. Following PCI, a significant increase in the level of prothrombin 1.2 fragments and thrombin-antithrombin complexes, markers of thrombin generation in circulating blood, were observed in the combined dabigatran group as compared to those immediately before the start of $\mathrm{PCl}$. In contrast, in the control group no such increase was noted. Moreover, 5 out of 40 patients required bailout anticoagulation in the dabigatran group, of whom four experienced a procedural MI, versus one out of 10 in the UFH group. One minor access-site bleeding occurred in the dabigatran group. Based on these preliminary results, dabigatran treatment provides an insufficient anticoagulation effect during $\mathrm{PCl}$ [14]. In a similar study, 108 stable CAD patients on DAPT undergoing elective PCI were randomized $(2: 2: 2: 1)$ to a short treatment course of rivaroxaban $10 \mathrm{mg}$, rivaroxaban $20 \mathrm{mg}$, rivaroxaban $10 \mathrm{mg}$ plus UFH or standard periprocedural UFH [15]. Rivaroxaban effectively suppressed blood coagulation activation as measured by prothrombin 1.2 fragments and thrombin-antithrombin complexes after elective $\mathrm{PCl}$ and stenting without an increased bleeding rate.

In the TAO trial [16], 13,229 patients were randomized either to a bolus of otamixaban, a synthetic intravenous direct factor $\mathrm{Xa}$ inhibitor, followed by one of two infusion doses of 0.100 or $0.140 \mathrm{mg} / \mathrm{kg} / \mathrm{h}$ or to UFH plus eptifibatide, at the time of $\mathrm{PCl}$. The final otamixaban infusion rate selected after interim analysis was

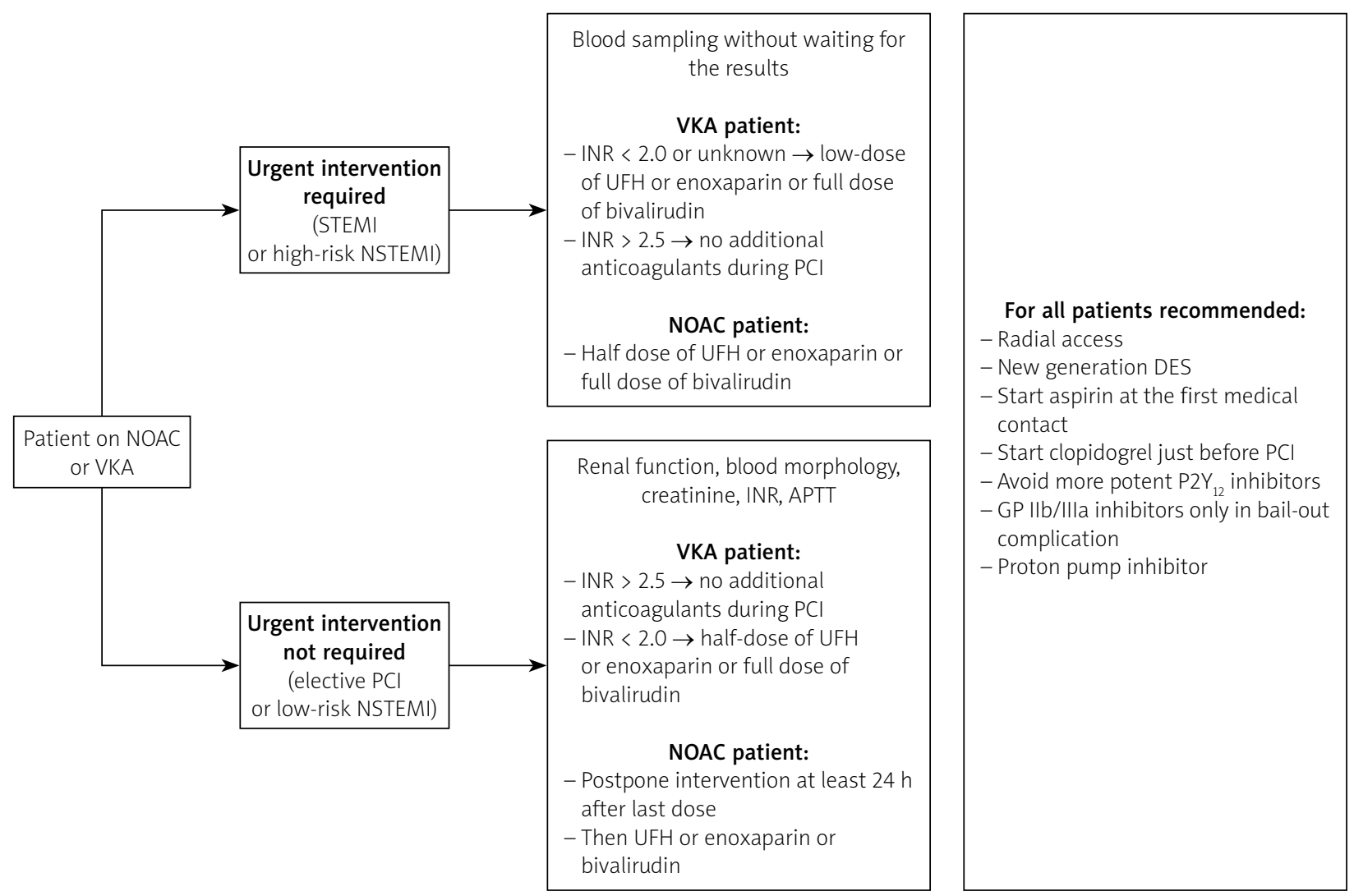

Figure 1. Periprocedural antithrombotic management 
$0.140 \mathrm{mg} / \mathrm{kg} / \mathrm{h}$. In the TAO trial $91 \%$ of patients received thienopyridine, mostly clopidogrel, $99 \%$ underwent coronary angiography, $65 \% \mathrm{PCl}$ and $29 \%$ were treated conservatively. The primary efficacy outcome expressed as death or MI by day 7 occurred in $5.5 \%$ of patients randomized to receive otamixaban and in $5.7 \%$ treated with UFH plus eptifibatide ( $p=0.93)$. Procedural thrombotic complications during the index $\mathrm{PCl}$ were found in $4.0 \%$ and $4.6 \%$, respectively. Prespecified subgroup analysis of the primary efficacy endpoint did not identify patients who benefited from otamixaban. The primary safety outcome of Thrombolysis in Myocardial Infarction (TIMI) major or minor bleeding by day 7 was significantly increased by otamixaban $(3.1 \%$ vs. $1.5 \%, p<0.001)$.

Because of lack of evidence for safety and efficacy of NOAC during $\mathrm{PCl}$, in elective patients it is recommended to discontinue NOAC at least $24 \mathrm{~h}$ before the procedure and to conduct periprocedural anticoagulation according to the local practice including UFH, enoxaparin or bivalirudin and a loading dose of DAPT with aspirin and clopidogrel (Figure 1) [17, 18]. Only in case of stent thrombosis while on clopidogrel, aspirin and OAC should more potent $\mathrm{P}_{2} \mathrm{Y}_{12}$ inhibitors, namely ticagrelor or prasugrel, be considered $[19,20]$. The management in very low risk ACS patients might be similar to elective procedures.

In STEMI or high risk NSTEMI patients to whom immediate $\mathrm{PCl}$ is strongly recommended regardless on the time elapsed since the last dose of VKA or NOAC, apart from a radial approach and loading dose of DAPT with aspirin and clopidogrel, it is recommended to use additional low-dose parental anticoagulation, i.e. UFH, enoxaparin or a full dose of bivalirudin. Because of uncertainty about the interpretation of routine coagulation tests in patients on NOAC, an anticoagulation strategy based on their results should not be used.

In patients with NSTEMI who do not require immediate intervention, the optimal time for the procedure might be established after measurement of routine coagulation tests, creatinine clearance and discontinuation of VKA or NOAC. In such ACS patients with delayed intervention of more than $12 \mathrm{~h}$ after the last dose of NOAC, fondaparinux or enoxaparin can be initiated. In all patients on OAC and DAPT, proton pump inhibitors should be promptly implemented $[1,17,18,21]$.

\section{Long-term treatment of patients on OAC after revascularization}

The oral anticoagulation should be started after elective $\mathrm{PCl}$ is completed or in the case of ACS after patient stabilization, if there is no vascular complication at the access site. In de novo AF the choice of anticoagulant depends on several factors. The candidates for warfarin therapy are patients unable to afford newer anticoagulants, with mechanical heart valves, creatinine clearance $<30 \mathrm{ml} / \mathrm{min}$, severe hepatic disease, and also those with frequent missed doses, multiple co-medications that may interact with NOAC, extremes of body weight, particularly those over $150 \mathrm{~kg}$, and patients with questionable adherence. In the majority of patients NOAC might be considered. Especially rivaroxaban or apixaban are preferred in patients with renal impairment or dyspepsia, apixaban or VKA after recent gastro-intestinal bleeding and dabigatran after ischemic stroke on warfarin [1, 22, 23]. In patients with $A F$ on anticoagulation before $P C l$, there is no evidence for benefits from switching to VKA or NOAC. Only in patients in whom ACS is caused by coronary artery embolization by thrombo-embolic material does an anticoagulant switch seem reasonable.

Currently it is unknown whether antiplatelet therapy with NOAC is safer and more effective in patients after $\mathrm{PCI}$ for ACS or stable CAD than antiplatelet therapy in combination with VKA. Because addition of any type of single antiplatelet therapy, especially DAPT, to VKA or NOAC anticoagulation significantly increases the risk of major bleeding, the European guidelines recommend maintaining the INR within 2.0-2.5 during triple or dual therapy with VKA and reducing doses of $\operatorname{NOAC}[17,18]$.

The effectiveness and safety of adding antiplatelet therapy to VKA in AF patients with stable CAD defined as at least 12 months from an ACS was examined in a large Danish registry [24]. Among 8700 patients followed for a median of 3.3 years, the risk of $\mathrm{MI}$, coronary death or thromboembolism was similar for VKA plus aspirin, VKA plus clopidogrel and VKA monotherapy, whereas the risk of bleeding significantly increased by 1.5 times when aspirin or by 1.84 times when clopidogrel was added to VKA.

A high risk of major bleeding with triple therapy in AF patients was also confirmed after acute MI [25] and following MI and coronary intervention [16]. In 40812 patients from the Danish nationwide registry during a mean follow-up of 477 days, $4.6 \%$ of patients were admitted to hospital due to bleeding complications [22]. With aspirin as reference, adjusted hazard ratios for bleeding were 1.33 for clopidogrel, 1.23 for VKA, 1.47 for aspirin plus clopidogrel, 1.84 for aspirin plus VKA, 3.52 for clopidogrel plus VKA, and 4.05 for triple therapy. There was a slightly higher bleeding risk with clopidogrel plus OAC than with aspirin plus OAC, as prior data had indicated [15]. In turn, in 11480 patients with AF who were admitted with MI or for $\mathrm{PCl}$, a $6.3 \%$ rate of bleeding events, including $0.7 \%$ fatal, was recorded within 1-year follow-up [26]. Bleeding risk with triple therapy exposure versus VKA plus one antiplatelet agent was significantly increased by 1.47 within the first 90 days and by 1.36 between 90 and 360 days. Simultaneously, no significant difference in thromboembolic risk was observed for triple therapy versus VKA combined with one antiplatelet agent.

A recent meta-analysis of seven randomized trials including ACS patients showed that the addition of NOAC to DAPT more than doubled the risk of major bleeding 
as compared to DAPT. On the other hand, adding NOAC to aspirin increases the major bleeding risk by $80 \%$ [27]. Simultaneously, NOAC reduced the incidence of MACE by $13 \%$ when added to DAPT and by $30 \%$ if added to aspirin.

To date, only two trials have randomly assigned patients requiring chronic anticoagulation and undergoing $\mathrm{PCl}$ to triple therapy versus dual therapy. In the WOEST trial [28], almost $70 \%$ of patients received OAC because of AF, but only a minority of patients had an ACS. Bleeding episodes were observed in $19.4 \%$ of patients receiving VKA and clopidogrel and in $44.4 \%$ of those receiving VKA, clopidogrel and aspirin continued for a whole year ( $p<0.0001)$. Simultaneously, the combined endpoint of death, MI, stroke, target-vessel revascularization and stent thrombosis was reported in $11.1 \%$ of patients in the dual therapy group and $17.6 \%$ in the triple therapy group ( $p=0.025)$, and all-cause mortality at 1 year was estimated at $2.5 \%$ vs. $6.3 \%(p=0.027)$, respectively. Although there are no data on how aspirin and VKA would have performed, dual therapy with VKA and clopidogrel seems to be safer and more efficient as compared with triple therapy. The investigators of the ISAR-TRIPLE study investigated whether shortening the duration of clopidogrel therapy from 6 months to 6 weeks after DES implantation is associated with a superior clinical outcome in 614 patients mostly with stable CAD (67\%) receiving concomitant aspirin and OAC [29]. The primary endpoint composed of death, MI, definite stent thrombosis, stroke, or TIMI major bleeding at 9 months occurred in $9.8 \%$ of patients in the 6-week group compared with $8.8 \%$ in the 6 -month group $(p=0.63)$. There were no significant differences in either major bleeding (5.3\% vs. $4.0 \%$, $p=0.44$ ) or thrombotic ( $4.0 \%$ vs. $4.3 \%, p=0.87$ ) events between the two strategies. Six weeks of triple therapy was comparable to 6 months with respect to net clinical outcomes.

The prospective, multicenter, observational AFCAS $(n=914)$ registry [30] demonstrated that at 1-year follow-up there was no difference among patients treated with triple therapy of VKA, aspirin, and clopidogrel versus DAPT vs. VKA plus clopidogrel in the propensity score-adjusted major adverse cardiac or cerebrovascular events ( $22 \%$ vs. $20 \%$ vs. $18 \%$, respectively, $p=0.72$ ). There was also no difference between groups regarding all-cause mortality ( $11 \%$ vs. $11 \%$ vs. $7 \%$, respectively, $p=0.54)$ or major bleeding ( $10 \%$ vs. $12 \%$ vs. $7 \%, p=0.43$ ).

The issue regarding optimal combination of anticoagulation with NOAC versus VKA with antiplatelet agents in AF patients after $\mathrm{PCl}$ with stenting will be hopefully provided by ongoing clinical trials (Table I). The time of combined antithrombotic and antiplatelet therapy after $\mathrm{PCl}$ depends on: i) indication for intervention (ACS, stable CAD), ii) stent type (preferred new-generation DES, BMS), iii) risk of bleeding (according to the HASBLED

Table I. Ongoing clinical trials regarding optimal combination of anticoagulation with NOAC versus VKA with antiplatelet agents in AF patients after PCI with stenting

\begin{tabular}{|c|c|c|c|}
\hline Trial acronym/status & $N$ & Trial aim/hypothesis & Study arms \\
\hline RE-DUAL PCI/recruiting & 2800 & $\begin{array}{l}\text { To study non-inferiority of each dose of dabiga- } \\
\text { tran arm when compared to warfarin in terms of } \\
\text { safety determined by major bleeding and clinical- } \\
\text { ly relevant non-major bleeding events according } \\
\text { to the modified ISTH classification }\end{array}$ & $\begin{array}{l}\text { 1. } 110 \text { mg dabigatran BID plus clopidogrel or tica- } \\
\text { grelor } \\
\text { 2. } 150 \mathrm{mg} \text { dabigatran BID plus clopidogrel or tica- } \\
\text { grelor } \\
\text { 3. A triple antithrombotic therapy of warfarin plus } \\
\text { clopidogrel or ticagrelor plus low-dose aspirin } \\
(<100 \mathrm{mg} \text { OD) }\end{array}$ \\
\hline $\begin{array}{l}\text { PIONEER AF PCI/study } \\
\text { completed }\end{array}$ & 2129 & $\begin{array}{l}\text { To evaluate the safety of three different treatment } \\
\text { strategies. } \\
\text { Safety in this trial is determined by significant } \\
\text { bleeding as a composite of TIMI major bleeding, } \\
\text { minor bleeding, and bleeding requiring medical } \\
\text { attention }\end{array}$ & $\begin{array}{l}\text { 1. } 15 \mathrm{mg} \text { rivaroxaban OD or } 10 \mathrm{mg} \text { for subjects } \\
\text { with moderate renal impairment plus clopido- } \\
\text { grel, prasugrel or ticagrelor } \\
\text { 2. } 2.5 \mathrm{mg} \text { rivaroxaban BID plus low-dose of aspirin } \\
\text { and clopidogrel, prasugrel or ticagrelor followed } \\
\text { by } 15 \mathrm{mg} \text { rivaroxaban OD plus low-dose aspirin } \\
\text { 3. VKA treatment strategy (target INR 2.0-3.0) } \\
\text { plus low-dose aspirin and clopidogrel, prasu- } \\
\text { grel or ticagrelor followed by VKA plus low-dose } \\
\text { aspirin for } 12 \text { months }\end{array}$ \\
\hline AUGUSTUS/recruiting & 4600 & $\begin{array}{l}\text { To determine whether apixaban is safer than VKA } \\
\text { given for } 6 \text { months in terms of bleeding in AF pa- } \\
\text { tients with ACS or } \mathrm{PCI} \text { with stent implantation } \\
\text { within the prior } 14 \text { days. The primary outcome } \\
\text { measure is time to first occurrence of major or } \\
\text { clinically relevant non-major bleeding according } \\
\text { to the ISTH classification }\end{array}$ & $\begin{array}{l}\text { Randomization in a } 2 \times 2 \text { factorial design to re- } \\
\text { ceive apixaban } 5 \mathrm{mg} O D \text { or } 2.5 \mathrm{mg} \mathrm{BID} \text {, with or } \\
\text { without aspirin, versus a VKA, with or without } \\
\text { aspirin. } \\
\text { All patients are receiving } \mathrm{P}_{2} \mathrm{Y}_{12} \text { inhibitors }\end{array}$ \\
\hline $\begin{array}{l}\text { EVOLVE AF PCI/accepted } \\
\text { by institutional board to } \\
\text { start recruitment }\end{array}$ & & Not determined yet & Treatment strategies with edoxaban are planned \\
\hline
\end{tabular}


score or other bleeding scores) and iv) risk of thromboembolic complications (according to the $\mathrm{CHA}_{2} \mathrm{DS}_{2}$-VASc score). Taking together available data, triple therapy should be kept as short as possible, 1 month after $\mathrm{PCl}$ for stable CAD and no longer than 6 months after an ACS. Then up to 12 months OAC and single antiplatelet therapy (SAPT) should be maintained. It remains unclear which would be that SAPT. The results of the WOEST trial suggest that clopidogrel might be preferred in the future. Currently there is no evidence to support the use of prasugrel or ticagrelor in combination with OAC. After 1 year following $\mathrm{PCl}$, in patients with stable vascular status defined as no recurrent ACS or no repeat revascularization, anticoagulation in monotherapy may be continued. Figure 2 summarizes previous recommendations $[1,15,16]$.
In the case of high risk of bleeding or current bleeding and implantation of new generation DES (Xience, Resolute) DAPT may be shortened to one month. It is worth remembering that in this group of patients left atrial appendage closure may also be considered [31]. In all patients on dual or triple therapy proton pump inhibitors should be implemented.

\section{Optimal stent management}

In the above-mentioned ISAR-TRIPLE trial [29], PCI with stenting in mostly stable CAD and AF patients with 6-week triple anticoagulation therapy with aspirin, clopidogrel and VKA was comparable with 6-month triple anticoagulation therapy with respect to net clinical outcomes. More than 90\% received new-generation DES (38.5\% everolimus-eluting stents (EES), 16.1\% biodegradable polymer

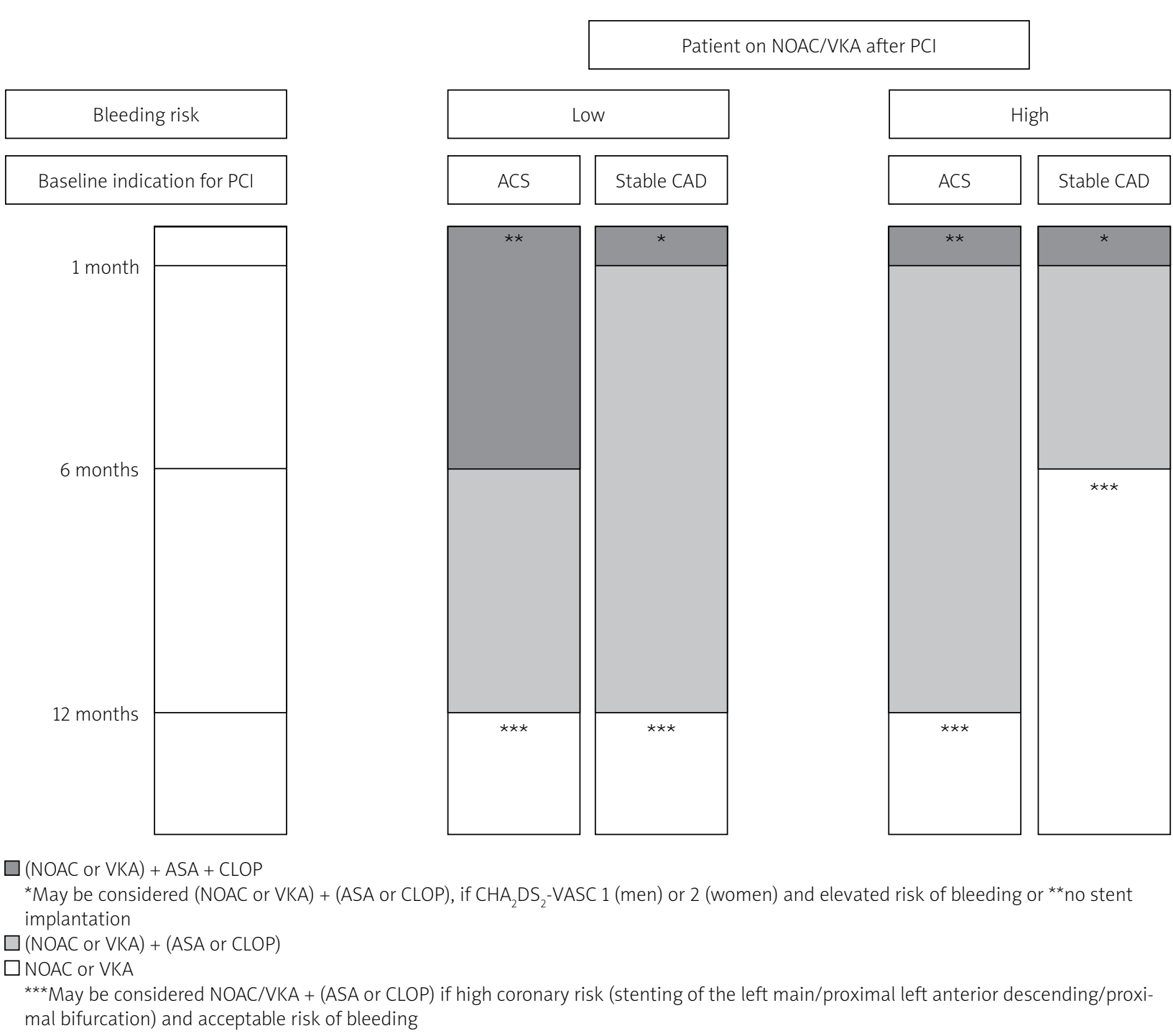

The dose of rivaroxaban 15 mg OD, apixaban 2.5 mg BID, edoxaban 30 mg OD or dabigatran 110 mg BID during triple/dual antithrombotic therapy according to the practical guide of the European Heart Rhythm Association [1]. It is not specified by the current atrial fibrillation guidelines of the ESC [17].

Figure 2. Long-term antithrombotic management 
Biolimus-eluting stents (BES), 16\% biodegradable polymer sirolimus-eluting stents (SES), $11 \%$ zotarolimus-eluting stents (ZES) and $11 \%$ probucol SES). However, the trial was not specifically powered to detect differences in the individual components of the primary endpoint.

The knowledge about appropriate stent choice comes from several trials evaluating short-term DAPT in patients without an indication for OAC. Two randomized trials, RESET (REal Safety and Efficacy of 3-month dual antiplatelet Therapy following Endeavor zotarolimus-eluting stent implantation) [32] and OPTIMIZE (Optimized Duration of Clopidogrel Therapy Following Treatment with the Zotarolimus-Eluting Stent in Real-World Clinical Practice) [33], evaluated 3 vs. 12 months of DAPT. In these two trials the prevalence of diabetes mellitus was $32 \%$ and $43 \%$ had ACS. In these two trials stent thrombosis was reported in $0.6 \%$ of patients with either 3- or 6-month DAPT, whereas clinically significant bleeding was reported in $0.6 \%$ and $0.9 \%$, respectively.

Five randomized trials have evaluated 6-month vs. either 12-month DAPT - ISAR-SAFE (Safety and Efficacy of Six Months Dual Antiplatelet Therapy After Drug-Eluting Stenting) [34], ITALIC (Is There A Life for DES After Discontinuation of Clopidogrel) [35], SECURITY (Second Generation Drug-Eluting Stent Implantation Followed by Six- Versus Twelve-Month Antiplatelet Therapy) [36], and EXCELLENT (Efficacy of Xience/Promus Versus Cypher to Reduce Late Loss After Stenting) [37] - or 24-month DAPT (PRODIGY (Prolonging Dual Antiplatelet Treatment After Grading Stent-Induced Intimal Hyperplasia Study)) [38]. Second-generation stents were used in all patients in the ITALIC and SECURITY trials. In the EXCELLENT and ISAR-SAFE trials, a second-generation DES was used in $75 \%$ and $88 \%$ of patients, respectively. In the PRODIGY trial, every $25 \%$ of patients received ZES, EES, BMS and paclitaxel-eluting stents (PES). $74.3 \%$ of patients had an ACS in the PRODIGY trial, 52\% in ISAR-SAVE and about $50 \%$ in EXCELLENT, ITALIC and SECURITY.

A meta-analysis of 6 randomized trials with shortterm DAPT (3 or 6 months) versus long-term DAPT (12 or 24 months) showed that compared with 12-or 24-month DAPT, short-term DAPT had an overall higher rate of stent thrombosis (odds ratio $(\mathrm{OR})=1.71, p=0.001$ ) [39]. The effect of short-term DAPT on stent thrombosis was attenuated with the use of second-generation DES as compared with the use of first-generation DES. Stent thrombosis rates with second-generation DES were $0.6 \%$ vs. $0.4 \%$, whereas with first-generation DES the rates were $2.4 \%$ vs. $0.6 \%$. There was a significant interaction between DES generation and DAPT treatment duration ( $p=0.008)$. Short-term DAPT had an overall lower risk of clinically significant bleeding $(\mathrm{OR}=0.63 ; p<0.001)$ and also a numerically lower all-cause mortality rate (2.0\% vs. $2.2 \%, p=0.073)$. It was found that for each stent thrombosis event averted by prolonging DAPT, an excess of 2.1 clinically significant bleeding events would be expected.
Recommendations for BMS implantation in patients who require triple therapy are lacking and differ between centers due to the paucity of reliable data. The current guidelines are inconsistent. According to the myocardial revascularization guidelines of the European Society of Cardiology (ESC) [40], new-generation DES are preferred over BMS in patients at low bleeding risk (HAS-BLED score $\leq 2$ ), whereas in high bleeding risk patients (HASBLED score $\geq 3$ ) the choice between BMS and new-generation DES needs to be decided on an individual basis. On the other hand, according to the practical guide of European Heart Rhythm Association [1] new generation DES or BMS are recommended to shorten the duration of the triple therapy. Recently, it was shown that in patients at high risk of bleeding or thrombosis, ZES combined with an abbreviated, tailored DAPT regimen resulted in a lower risk of 1-year death, MI or target vessel revascularization as compared with BMS [41]. Undoubtedly bioresorbable stents should be avoided in patients requiring OAC due to the need for prolonged DAPT [42, 43].

The optimal duration of DAPT with second-generation DES outside ACS still remains to be established. However, the timing of interruption of a thienopyridine can be as short as 3 or 6 months after stent implantation. The results indicate that in the case of ZES clopidogrel discontinuation may be safely done after 3-month treatment with DAPT.

\section{Bleeding management}

Bleeding complications remain an important issue during long-term OAC. The results of a prespecified meta-analysis of all 71,683 AF patients included in the RE-LY, ROCKET AF, ARISTOTLE, and ENGAGE AF-TIMI 48 trials [44] showed that as compared with warfarin, NOAC significantly reduced events of intracranial hemorrhage from $0.66 \%$ to $0.32 \%(p<0.0001)$, but increased gastrointestinal bleeding from $0.92 \%$ to $1.17 \%$ ( $p=0.04)$. Moreover, in comparison to warfarin, fatal bleeding risk was reduced with all NOAC. The reduction with apixaban was from $0.19 \%$ to $0.16 \%$, with dabigatran from $0.3 \%$ to $0.21 \%$, with edoxaban from $0.84 \%$ to $0.38 \%$, and with rivaroxaban from $0.77 \%$ to $0.38 \%$ [45]. Also case fatality due to major bleeding was reduced with dabigatran (3.37\% vs. $4.23 \%)$, edoxaban (7.74\% vs. $11.23 \%)$ and rivaroxaban (6.84\% vs. $14.25 \%)$ but not with apixaban (4.59\% vs. $3.68 \%$ ) as compared to warfarin.

As expected, concomitant antiplatelet drugs increased the risk of major bleeding in AF patients receiving dabigatran as compared to those without antiplatelet agents (4.4\% vs. $2.6 \%$ ), irrespective of the dabigatran dose [46]. In those who used antiplatelet agents, the absolute risk of bleeding was lowest with dabigatran $110 \mathrm{mg}$ twice a day (BID), followed by dabigatran $150 \mathrm{mg}$ BID and warfarin, with rates of $3.9 \%, 4.4 \%$, and $4.8 \%$ per year, respectively. Also the risk of major bleeding was higher among 
patients who received dual antiplatelets $(H R=2.31)$ than among patients who only received a single antiplatelet $(H R=1.60)$ ( $p$ for trend $<0.001)$ in comparison to patients without antiplatelet therapy. A recently published subanalysis of 14,171 patients enrolled in the ROCKET-AF trial [47] showed that $\mathrm{PCl}$ occurred infrequently in $1.1 \%$ of patients with AF during a median 806 days of follow-up. DAPT of more than 30 days was used in $37 \%$, single antiplatelet therapy in 34\%, and the majority of patients (81\%) remained on anticoagulation after $\mathrm{PCl}$. Rates of both thrombotic and bleeding events were high in the post- $\mathrm{PCl}$ period, and most of them occurred within 6 months. In patients on rivaroxaban versus warfarin, rates of stroke/systemic embolism were $5.0 \%$ vs. $4.1 \% / 100$ patient-years and major bleeding events $15.0 \%$ vs. $8.1 / 100$ patient-years, respectively.

In patients with bleeding during OAC it is necessary to determine in what circumstances the event occurred, how much time elapsed since the intake of the last dose of anticoagulant agent, whether the patient suffers from chronic kidney disease and which concomitant drugs are taken concomitantly. It is also recommended to sample the patient for blood cell counts, serum creatinine, prothrombin time (PT) or INR, if he or she is on VKA, and activated partial thromboplastin time (APTT). Regarding NOAC, rivaroxaban can prolong PT (and increase the automatically calculated INR) and APTT, whereas dabigatran increases APTT, thrombin time (TT), and ecarin clotting time, with a slight increase in PT-INR at high concentrations and with a weak correlation with their plasma concentration measured directly by the high-performance liquid chromatography (HPLC)-tandem mass spectrometry [48-53]. Currently, routine laboratory monitoring of NOAC concentrations using clot-based assays is not recommended [50, 54]. However, there are clinical situations including bleeding complications in which measurements of the anticoagulant effect of NOAC are desirable. The most common tests used for this purpose are calibrated quantitative anti-factor-Xa assays for factor Xa inhibitors or the Hemoclot assays measuring dilute TT in patients on dabigatran. These tests may not be readily available, but in some labs they are performed 24/7 and their results are available up to $2 \mathrm{~h}[51,52,55]$.

In patients with minor bleeding usually local compression is enough and the next doses of anticoagulant should be omitted depending on the bleeding intensity and patient status. In patients with normal renal function, hemostasis normalizes within 12-24 h after the last dose of NOAC. This time can be prolonged even more than $48 \mathrm{~h}$ in patients with severe chronic kidney disease. Patients with moderate to severe bleeding require additionally blood pressure monitoring, diuresis maintenance and, depending on patient status and laboratory parameters, fluid replacement, plasma, platelet or blood transfusion and more invasive procedures, e.g. gastroscopy, colonoscopy or bronchoscopy. In patients who received NOAC within the last 2-4 h charcoal administration and/ or gastric lavage might be considered. Moreover, dabigatran can be dialyzed, but there is still limited experience with this approach.

In patients with life-threatening bleeding, reversal of the antithrombotic effect is indicated. In subjects on VKA, prothrombin complex concentrate (PCC) or, if unavailable, fresh frozen plasma and/or vitamin $\mathrm{K}$, that require $24-48 \mathrm{~h}$ to achieve normal blood coagulation, should be administered in a dose adjusted to the INR value. In terms of bleeding complications associated with dabigatran, it was shown that idarucizumab, a humanized antibody fragment which irreversibly binds dabigatran, administered as an intravenous dual bolus of $2.5 \mathrm{~g}$, normalized elevated dilute TT and elevated ecarin clotting time in $88 \%$ to $98 \%$ of patients with gastrointestinal bleeding, intracranial hemorrhage, trauma or requiring immediate surgery within minutes after antidote administration [56]. Simultaneously, one thrombotic event was reported within $72 \mathrm{~h}$ after idarucizumab administration in a patient to whom anticoagulants had not been reinitiated. Recently, it has been reported that andexanet alfa, an inactive recombinant human factor $\mathrm{Xa}$ which binds factor $\mathrm{Xa}$ inhibitors, administered as a bolus or as a bolus plus a 2-hour infusion, in healthy older volunteers receiving either $5 \mathrm{mg}$ of apixaban twice daily or $20 \mathrm{mg}$ of rivaroxaban daily, reduced anti-factor Xa activity by $94 \%$ or $92 \%$, within 2 to 5 min after antidote administration, respectively [57]. Simultaneously, a transient increase of D-dimer and prothrombin 1.2 fragments was observed, without serious adverse or thrombotic events. Until October 2016 solely idarucizumab has been approved by the US Food and Drug Administration and the European Medicines Agency and is increasingly used worldwide [58]. Andexanet alfa is expected to be approved for clinical use at the end of 2017. However, the experience with both idarucizumab and andexanet alfa in patients with ACS and/or shortly after stent implantation is very limited.

If a specific antidote is not available, PCC, preferably 30-50 U/kg body weight with another half dose if the effect is suboptimal, should be promptly administered in patients on NOAC with life-threatening bleeding. The proportion of patients who need such therapy due to severe bleeding is about $5-10 \%$ of those with major bleeding, mostly elderly individuals with renal impairment.

\section{ACS as a primary indication for NOAC}

Tremendous progress in invasive and pharmacological therapy of $\mathrm{Ml}$ is associated with reduction of 30-day mortality to $7.8 \%$ [59] and $12-13 \%$ after 6 months [60, $61]$. Despite high rates of implementation of the guideline recommended therapy including aspirin, clopidogrel up to 1 year after an ACS event [62] and statin, at 5 years $19 \%$ of patients with STEMI died, $22 \%$ with NSTEMI and $17 \%$ with unstable angina (UA) [63]. 
Because DAPT, even with the use of more potent $\mathrm{P}_{2} \mathrm{Y}_{12}$ inhibitors $[64,65]$, is insufficient to abolish the residual risk of long-term recurrence, there is clinical evidence for implementation of antithrombin strategies, targeting both attenuation of platelet activation and aggregation as well as suppression of thrombin generation and/or thrombin activity. Therefore, selective anticoagulants, direct thrombin inhibitors or factor $\mathrm{Xa}$ inhibitors, have been tested in the ACS population.

\section{Direct thrombin inhibitors}

In the phase II, dose-escalation RE-DEEM trial, 1861 STEMI (60\%) and NSTEMI (40\%) patients on DAPT were randomized within 14 days after the index event to treatment with dabigatran in a dose of 50-150 mg BID, or placebo [66]. The primary outcome measure defined as the composite of major or clinically relevant minor bleeding during the 6-month treatment period occurred in 3.5, 4.3, 7.9 and $7.8 \%$ in the respective 50, 75, 110 and $150 \mathrm{mg}$ BID dabigatran groups ( $p<0.001$ for trend), compared with $2.2 \%$ in the placebo group. Female gender and age $>75$ years were associated with a higher rate of bleeding in patients receiving the two highest dabigatran doses.

In terms of efficacy issues, death, $\mathrm{Ml}$ or stroke occurred in $4.6 \%$ of patients treated with $50 \mathrm{mg}$ of dabigatran, in $4.9 \%$ with $75 \mathrm{mg}, 3.0 \%$ with $110 \mathrm{mg}$, and 3.5\% with a dabigatran dose of $150 \mathrm{mg}$ as compared with $3.5 \%$ in the placebo group [48]. In patients receiving dabigatran, median D-dimer levels were $37 \%$ and $45 \%$ lower at weeks 1 and 4, respectively, as compared with patients in the placebo group $(p<0.001)$.

\section{Direct Xa inhibitors}

The safety and efficacy of apixaban administered up to 7 days after either STEMI or moderate- or high-risk NSTEMI/UA have been tested in the two APPRAISE trials $[67,68]$. The phase II APPRAISE study compared four apixaban doses of $5 \mathrm{mg}$ twice daily, $10 \mathrm{mg}$ once daily, $10 \mathrm{mg}$ twice daily or $20 \mathrm{mg}$ once daily with placebo [59]. The enrolment to the two highest doses of apixaban was discontinued because of an excessive rate of bleeding. Of the 1715 patients randomized, 65\% underwent PCI before enrolment, $76 \%$ received clopidogrel and aspirin, $65 \%$ were diagnosed with STEMI, 30\% with NSTEMI and $8 \%$ with UA. Over a 6 -month treatment, the primary outcome measure expressed as major or clinically relevant non-major bleeding according to the ISTH was found in $5.7 \%$ of patients with apixaban $2.5 \mathrm{mg}$ twice daily ( $p=$ 0.09 ) and in $7.9 \%$ with apixaban $10 \mathrm{mg}$ once daily ( $p=$ $0.005)$ vs. $3.0 \%$ in the placebo group.

A dose-related increase in bleeding and a trend toward a reduction in ischemic events with the addition of apixaban to antiplatelet therapy in patients with a recent ACS was verified in the APPRAISE-2 study [68]. In this study 7392 patients were randomly assigned to receive either apixaban at a dose of $5 \mathrm{mg}$ twice daily or placebo. The primary safety outcome expressed as TIMI major bleeding occurred in $1.3 \%$ of patients who received apixaban and in $0.5 \%$ of patients with placebo $(p=0.001)$. Importantly, intracranial bleeding $(0.3 \%$ vs. $0.1 \%, p=$ $0.03)$ and fatal bleeding $(0.1 \%$ vs. $0 \%)$ were more frequent in patients receiving apixaban as compared with placebo. During a median 6-month follow-up, the primary outcome of cardiovascular death, MI, or ischemic stroke occurred in $7.5 \%$ of patients receiving apixaban and in $7.9 \%$ assigned to placebo $(p=0.51)$. Net clinical outcome expressed as a composite endpoint of cardiovascular death, MI, ischemic or hemorrhagic stroke or fatal bleeding was found in $8.0 \%$ of the apixaban group and in $8.1 \%$ of the placebo group $(p=0.80)$.

In both APPRAISE trials an increase in bleeding with apixaban was evident immediately after randomization. The addition of apixaban to antiplatelet therapy in high risk patients after an ACS significantly increased bleeding complications, without a significant reduction in recurrent ischemic events.

In the ATLAS ACS-TIMI 46 phase II trial, 3491 patients stratified according to the use of aspirin or aspirin plus thienopyridine, 1-7 days after hospital admission for an ACS were randomized to placebo or rivaroxaban at doses of 5-20 mg given once or at the same total daily dose twice [69]. After 6-month follow-up, of 299 clinically significant bleeding events, $83 \%$ were categorized as requiring medical attention, $11 \%$ as TIMI major, and $6 \%$ as TIMI minor bleeding. All those bleeding events occurred in a dose-dependent manner with hazard ratios of 2.2 for $5 \mathrm{mg}, 3.4$ for $10 \mathrm{mg}, 3.6$ for $15 \mathrm{mg}$, and 5.1 for $20 \mathrm{mg}$ rivaroxaban daily doses $(p<0.0001)$ as compared to placebo. In the whole cohort, rates of the primary efficacy endpoint defined as death, $\mathrm{MI}$, stroke, or severe recurrent ischemia requiring revascularization were $5.6 \%$ for rivaroxaban and $7.0 \%$ for placebo (relative risk reduction of $21 \%, p=0.10$ ). By exploratory analysis, a significant reduction in primary efficacy endpoint with rivaroxaban was observed in patients aged above 65 years by $53 \%$ ( $p$ for interaction $=0.007$ ) and in patients with STEMI by $42 \%$ ( $p$ for interaction $=0.042$ ).

The results of the ATLAS ACS-TIMI 46 trial allowed selection of the most favorable dose and dosing regimen of rivaroxaban in combination with aspirin or aspirin and thienopyridine, which was applied in the phase III ATLAS ACS2-TIMI 51 study [70]. This trial was designed to test whether inhibition of factor Xa with low-dose rivaroxaban might improve cardiovascular outcomes in patients with a recent ACS. 15526 ACS patients were randomly assigned to twice-daily administration of either $2.5 \mathrm{mg}$ or $5 \mathrm{mg}$ of rivaroxaban or placebo with a mean follow-up period of 12 months. Rivaroxaban significantly reduced the primary efficacy endpoint of death from cardiovascular causes, MI, or stroke as compared with placebo (8.9\% vs. $10.7 \%, p=0.008)$. For both the twice-daily 2.5 -mg dose 
(9.1\% vs. $10.7 \%, p=0.02)$ and the twice-daily 5 -mg dose (8.8\% vs. $10.7 \%, p=0.03$ ) significant improvement of the primary efficacy endpoint was found. The lower dose of rivaroxaban but not twice-daily $5 \mathrm{mg}$ also reduced the rates of death from cardiovascular causes (2.7\% vs. $4.1 \%)$ and from any cause ( $2.9 \%$ vs. $4.5 \%, p=0.002$ for both). Rivaroxaban also reduced the risk of in-stent thrombosis as compared with placebo $(2.3 \%$ vs. $2.9 \%, p=0.02)$.

Compared with placebo, both rivaroxaban doses increased 3.5-4.5 times the risk of major bleeding not related to CABG ( $p<0.001$ for both) and 2.8-3.8 times the risk of intracranial hemorrhage ( $p<0.05$ for both), without a significant increase in fatal bleeding $(0.3 \% \mathrm{vs}$. $0.2 \%)$. In comparison of two rivaroxaban doses, significantly lower rates of TIMI minor bleeding (0.9\% vs. $1.6 \%$, $p<0.05)$, TIMI bleeding requiring medical attention (12.9\% vs. $16.2 \%, p<0.001)$ and fatal bleeding $(0.1 \%$ vs. $0.4 \%, p=0.04$ ) were found with the lower dose [70].

\section{DAPT versus SAPT with NOAC after ACS}

Although in the APPRAISE trial [67] patients receiving DAPT were younger and less frequently had renal insufficiency or cerebrovascular disease than patients receiving aspirin alone, the dose-related increase of bleeding with apixaban was more apparent in patients taking DAPT. As compared with placebo, among patients receiving clopidogrel and aspirin the adjusted rate difference in major or clinically relevant bleeding was $2.2 \%$ for apixaban $2.5 \mathrm{mg}$ BID and 3.8\% for apixaban $10 \mathrm{mg}$ once daily (OD) and in patients on aspirin $-0.7 \%$ and $0.8 \%$, respectively. The incidence of cardiovascular death, $\mathrm{MI}$, severe recurrent ischemia, or ischemic stroke was not significantly lower in patients receiving apixaban $2.5 \mathrm{mg}$ BID (7.6\%, $p=0.21)$ and $10 \mathrm{mg} \mathrm{OD}(6.0 \%, p=0.07)$ as compared with placebo (8.0\%). Although patients on both DAPT and aspirin alone tended to have lower rates of ischemic events with both apixaban doses, the incidence of composite ischemic endpoint was about twice as low on DAPT.

In ATLAS ACS-TIMI 46 [69], across all rivaroxaban doses (5-20 mg), the absolute rate of clinically significant bleeding was lower in patients receiving aspirin only (2.9-10.7\%) than in patients on DAPT $(11.7-16.0 \%, p<$ $0.0001)$. In turn, compared with placebo, in patients with aspirin alone rivaroxaban reduced the risk of primary efficacy endpoint by $47 \%$, but only by $1 \%$ in patients on DAPT ( $p$ for interaction $=0.034$ ). In patients treated with aspirin only but not on DAPT, a significant reduction of death, $\mathrm{MI}$ and stroke with 5,10 or $20 \mathrm{mg}$ of rivaroxaban versus placebo was found ( $8.0 \%, 7.0 \%$ or $4.7 \%$ vs. $11.9 \%$, respectively, $p=0.01$ for trend).

The net clinical outcome associated with rivaroxaban defined as hazard ratio of death, MI, stroke, or TIMI major bleeding, as compared with placebo in patients on aspirin only, was 0.57 , and on DAPT 1.17. The respective hazard ratios assessed in participants with a dose of
$2.5 \mathrm{mg}$ or $5 \mathrm{mg}$ twice daily were 0.59 in patients with aspirin and 0.85 in patients on DAPT [70].

\section{Future perspectives for NOAC after ACS}

Currently NOAC are not recommended for treatment of the acute phase of ACS. The role of NOAC in combination with more potent DAPT in secondary prevention after ACS is promising but requires further research. The safety and efficacy of rivaroxaban as compared with aspirin, in addition to a single antiplatelet $\mathrm{P}_{2} \mathrm{Y}_{12}$ inhibitor clopidogrel or ticagrelor - in subjects with a recent STEMI or NSTEMI are being tested in the ongoing GEMINI trial. There is also no evidence that prothrombotic genetically determined factors might affect the efficacy or safety of NOAC used in monotherapy or combination [71].

\section{Summary}

Available, mostly observational data indicate that in patients who require oral anticoagulation, both VKA and NOAC could be used with aspirin and clopidogrel following $\mathrm{PCl}$ in $\mathrm{MI}$ and stable angina patients, but the optimal combination of anticoagulation with NOAC versus VKA with antiplatelet agents will hopefully be provided by ongoing clinical trials. The safety and efficacy of prasugrel and ticagrelor taken with oral anticoagulants also remain to be established in randomized trials; therefore the $\mathrm{P}_{2} \mathrm{Y}_{12}$ inhibitor clopidogrel on top of aspirin or without is now recommended to be used together with a VKA or NOAC. It is still unclear which dose of a NOAC in combination with antiplatelet agents and different stents should be used in this clinical setting and whether indeed NOAC are safer compared with VKA in such cardiovascular patients. Another controversial issue is whether or not to use oral anticoagulation in addition to antiplatelet therapy for secondary prevention in patients with ACS. The safety and efficacy of prasugrel and ticagrelor taken with NOAC also remain to be established in randomized trials. To minimize bleeding risk, a tailored approach should be implemented in which the right agent will be prescribed to the right patient at the right dose and supported by regular clinical evaluation and laboratory testing, especially renal function. It is now unclear whether the regular full dose NOAC are safe as compared to previously recommended reduced doses of these agents. Observational studies and ongoing randomized controlled trials are likely to optimize dosage and duration of this complex antithrombotic therapy in patients following $\mathrm{PCl}$.

\section{Conflict of interest}

The authors declare no conflict of interest.

\section{References}

1. Lip GY, Windecker S, Huber K, et al. Management of antithrombotic therapy in atrial fibrillation patients presenting with acute coronary syndrome and/or undergoing percutaneous coronary 
or valve interventions: a joint consensus document of the European Society of Cardiology Working Group on Thrombosis, European Heart Rhythm Association (EHRA), European Association of Percutaneous Cardiovascular Interventions (EAPCI) and European Association of Acute Cardiac Care (ACCA) endorsed by the Heart Rhythm Society (HRS) and Asia-Pacific Heart Rhythm Society (APHRS). Eur Heart J 2014; 35: 3155-79.

2. Kralev S, Schneider K, Lang S, et al. Incidence and severity of coronary artery disease in patients with atrial fibrillation undergoing first-time coronary angiography. PloS One 2011; 6: e24964.

3. Lopes RD, Pieper KS, Horton JR, et al. Short- and long-term outcomes following atrial fibrillation in patients with acute coronary syndromes with or without ST-segment elevation. Heart 2008; 94: 867-73.

4. Rene AG, Genereux P, Ezekowitz M, et al. Impact of atrial fibrillation in patients with ST-elevation myocardial infarction treated with percutaneous coronary intervention (from the HORIZONS-AMI [Harmonizing Outcomes With Revascularization and Stents in Acute Myocardial Infarction] trial). Am J Cardiol 2014; 113: 236-42.

5. Dzeshka MS, Brown RA, Lip GY. Patients with atrial fibrillation undergoing percutaneous coronary intervention. Current concepts and concerns: part I. Pol Arch Med Wewn 2015; 125: 73-81.

6. Dzeshka MS, Brown RA, Lip GY. Patients with atrial fibrillation undergoing percutaneous coronary intervention: current concepts and concerns: part II. Pol Arch Med Wewn 2015; 125: 172-80.

7. Gurbel PA, Navarese EP, Tantry US. What is the best anticoagulant therapy during primary percutaneous coronary intervention for acute myocardial infarction? Pol Arch Med Wewn 2015; 125 : 461-70.

8. Niewada M, Członkowska A. Prevention of ischemic stroke in clinical practice: a role of internists and general practitioners. Pol Arch Med Wewn 2014; 124: 540-8.

9. Trusz-Gluza M, Filipecki A, Urbańczyk-Świć D. Patients with atrial fibrillation and low risk of stroke: do they really Reed anticoagulation? Pol Arch Med Wewn 2015; 125: 678-84.

10. Andó G, Costa F. Bleeding risk stratification in acute coronary syndromes. Is it still valid in the era of the radial approach? Postep Kardiol Interw 2015; 11: 170-3.

11. Lahtela H, Rubboli A, Schlitt A, et al. Heparin bridging vs. uninterrupted oral anticoagulation in patients with Atrial Fibrillation undergoing Coronary Artery Stenting. Results from the AFCAS registry. Circ J 2012; 76: 1363-8.

12. Dewilde WJ, Janssen PW, Kelder JC, et al. Uninterrupted oral anticoagulation versus bridging in patients with long-term oral anticoagulation during percutaneous coronary intervention: subgroup analysis from the WOEST trial. Eurolntervention 2015; 11: 381-90.

13. Atas H, Sari I, Delil K, et al. Acute inferior myocardial infarction in a patient with a prosthetic aortic valve and high international normalized ratio. Postep Kardiol Interw 2014; 10: 63-5.

14. Vranckx P, Verheugt FW, de Maat MP, et al. A randomised study of dabigatran in elective percutaneous coronary intervention in stable coronary artery disease patients. Eurolntervention 2013; 8: 1052-60.

15. Vranckx P, Leebeek FW, Tijssen J, et al. Periprocedural use of rivaroxaban in elective percutaneous coronary intervention to treat stable coronary artery disease. The X-PLORER trial. Thromb Haemost 2015; 114: 258-67.

16. Steg PG, Mehta SR, Pollack CV Jr, et al. Anticoagulation with otamixaban and ischemic events in non-ST-segment elevation acute coronary syndromes: the TAO randomized clinical trial. JAMA 2013; 310: 1145-55.

17. Kirchhof P, Benussi S, Kotecha D, et al. 2016 ESC Guidelines for the management of atrial fibrillation developed in collaboration with EACTS: The Task Force for the management of atrial fibrillation of the European Society of Cardiology (ESC) Developed with the special contribution of the European Heart Rhythm Association (EHRA) of the ESC Endorsed by the European Stroke Organisation (ESO). Eur Heart J 2016 Aug 27. pii: ehw210.

18. Heidbuchel $H$, Verhamme P, Alings $M$, et al. Updated European Heart Rhythm Association Practical Guide on the use of non-vitamin $\mathrm{K}$ antagonist anticoagulants in patients with non-valvular atrial fibrillation. Europace 2015; 17: 1467-507.

19. Winter MP, Koziński M, Kubica J, et al. Personalized antiplatelet therapy with $\mathrm{P} 2 \mathrm{Y} 12$ receptor inhibitors: benefits and pitfalls. Postep Kardiol Interw 2015; 11: 259-80.

20. Komosa A, Lesiak M, Siniawski A, et al. Significance of antiplatelet therapy in emergency myocardial infarction treatment. Postep Kardiol Interw 2014; 10: 32-9.

21. Kwiecień S, Magierowska K, Śliwowski Z, et al. New insight into the mechanisms of gastroduodenal injury induced by nonsteroidal anti-inflammatory drugs: practical implications. Pol Arch Med Wewn 2015; 125: 191-8.

22. Kitchen S, Gray E, Mackie I, et al.; BCSH committee. Measurement of non-coumarin anticoagulants and their effects on tests of haemostasis: guidance from the British Committee for Standards in Haematology. Br J Haematol 2014; 166: 830-41.

23. Zielonka A, Tkaczyszyn M, Mende $M$, et al. Atrial fibrillation in outpatients with stable coronary artery disease: results from the multicenter RECENT study. Pol Arch Med Wewn 2015; 125: 162-71.

24. Lamberts M, Gislason GH, Lip GY, et al. Antiplatelet therapy for stable coronary artery disease in atrial fibrillation patients taking an oral anticoagulant: a nationwide cohort study. Circulation 2014; 129: 1577-85.

25. Sorensen R, Hansen ML, Abildstrom SZ, et al. Risk of bleeding in patients with acute myocardial infarction treated with different combinations of aspirin, clopidogrel, and vitamin $\mathrm{K}$ antagonists in Denmark: a retrospective analysis of nationwide registry data. Lancet 2009; 374: 1967-74.

26. Lamberts $M$, Olesen JB, Ruwald $M H$, et al. Bleeding after initiation of multiple antithrombotic drugs, including triple therapy, in atrial fibrillation patients following myocardial infarction and coronary intervention: a nationwide cohort study. Circulation 2012; 126: 1185-93.

27. Oldgren J, Wallentin L, Alexander JH, et al. New oral anticoagulants in addition to single or dual antiplatelet therapy after an acute coronary syndrome: a systematic review and meta-analysis. Eur Heart J 2013; 34: 1670-80.

28. Dewilde WJ, Oirbans T, Verheugt FW, et al. Use of clopidogrel with or without aspirin in patients taking oral anticoagulant therapy and undergoing percutaneous coronary intervention: an open-label, randomised, controlled trial. Lancet 2013; 381: 1107-15.

29. Fiedler KA, Maeng M, Mehilli J, et al. Duration of triple therapy in patients requiring oral anticoagulation after drug-eluting stent implantation: the ISAR-TRIPLE Trial. J Am Coll Cardiol 2015; 65: 1619-29.

30. Rubboli A, Schlitt A, Kiviniemi T, et al. One-year outcome of patients with atrial fibrillation undergoing coronary artery stenting: an analysis of the AFCAS registry. Clin Cardiol 2014; 37: 357-64. 
31. Pracoń R, Demkow M. How to walk the tightrope between harm and protection in selecting the optimal antiplatelet treatment strategy after transcatheter left atrial appendage occlusion. Postep Kardiol Interw 2015; 11: 5-8.

32. Kim BK, Hong MK, Shin DH, et al. A new strategy for discontinuation of dual antiplatelet therapy: the RESET Trial (REal Safety and Efficacy of 3-month dual antiplatelet Therapy following Endeavor zotarolimus-eluting stent implantation). J Am Coll Cardiol 2012; 60: 1340-8.

33. Feres F, Costa RA, Abizaid A, et al. Three vs twelve months of dual antiplatelet therapy after zotarolimus-eluting stents: the OPTIMIZE randomized trial. JAMA 2013; 310: 2510-22.

34. Schulz-Schupke S, Byrne RA, Ten Berg JM, et al. ISAR-SAFE: a randomized, double-blind, placebo-controlled trial of 6 versus 12 months of clopidogrel therapy after drug-eluting stenting. Eur Heart J 2015; 36: 1252-63.

35. Gilard M, Barragan P, Noryani AA, et al. Six-month versus 24-month dual antiplatelet therapy after implantation of drug eluting stents in patients non-resistant to aspirin: ITALIC, a randomized multicenter trial. J Am Coll Cardiol 2015; 65: 777-86.

36. Colombo A, Chieffo A, Frasheri A, et al. Second-generation drug-eluting stent implantation followed by 6-versus 12-month dual antiplatelet therapy: the SECURITY randomized clinical trial. J Am Coll Cardiol 2014; 64: 2086-97.

37. Gwon HC, Hahn JY, Park KW, et al. Six-month versus 12-month dual antiplatelet therapy after implantation of drug-eluting stents: the Efficacy of Xience/Promus Versus Cypher to Reduce Late Loss After Stenting (EXCELLENT) randomized, multicenter study. Circulation 2012; 125: 505-13.

38. Valgimigli $M$, Campo G, Monti $M$, et al. Short- versus long-term duration of dualantiplatelet therapy after coronary stenting: a randomized multicenter trial. Circulation 2012; 125: 2015-26.

39. Giustino G, Baber U, Sartori S, et al. Duration of dual antiplatelet therapy after drug-eluting stent implantation: a systematic review and meta-analysis of randomized controlled trials. J Am Coll Cardiol 2015; 65: 1298-310.

40. Windecker S, Kolh P, Alfonso F, et al. 2014 ESC/EACTS Guidelines on myocardial revascularization: The Task Force on Myocardial Revascularization of the European Society of Cardiology (ESC) and the European Association for Cardio-Thoracic Surgery (EACTS) developed with the special contribution of the European Association of Percutaneous Cardiovascular Interventions (EAPCI). Eur Heart J 2014; 35: 2541-619.

41. Valgimigli $M$, Patialiakas A, Thury A, et al. Zotarolimus-eluting versus bare-metal stents in uncertain drug-eluting stent candidates. J Am Coll Cardiol 2015; 65: 805-15.

42. Giacchi G, Ortega-Paz L, Brugaletta S, et al. Bioresorbable vascular scaffold implantation in acute coronary syndromes: clinical evidence, tips and tricks. Postep Kardiol Interw 2015; 11: 161-9.

43. Lesiak M, Araszkiewicz A. "Leaving nothing behind": is the bioresorbable vascular scaffold a new hope for patients with coronary artery disease? Postep Kardiol Interw 2014; 10: 283-8.

44. Ruff CT, Giugliano RP, Braunwald E, et al. Comparison of the efficacy and safety of new oral anticoagulants with warfarin in patients with atrial fibrillation: a meta-analysis of randomised trials. Lancet 2014; 383: 955-62.

45. Caldeira D, Rodrigues FB, Barra M, et al. Non-vitamin K antagonist oral anticoagulants and major bleeding-related fatality in patients with atrial fibrillation and venous thromboembolism: a systematic review and meta-analysis. Heart 2015; 101: 1204-11.
46. Dans AL, Connolly SJ, Wallentin L, et al. Concomitant use of antiplatelet therapy with dabigatran or warfarin in the Randomized Evaluation of Long-Term Anticoagulation Therapy (RE-LY) trial. Circulation 2013; 127: 634-40.

47. Sherwood MW, Cyr DD, Jones WS, et al. Use of dual antiplatelet therapy and patient outcomes in those undergoing percutaneous coronary intervention: the ROCKET AF Trial. JACC Cardiovasc Interv 2016; 9: 1694-702.

48. Douxfils J, Dogné JM, Mullier F, et al. Comparison of calibrated dilute thrombin time and aPTT tests with LC-MS/MS for the therapeutic monitoring of patients treated with dabigatran etexilate. Thromb Haemost 2013; 110: 543-9.

49. Hillarp A, Baghaei F, Fagerberg Blixter I, et al. Effects of the oral, direct factor Xa inhibitor rivaroxaban on commonly used coagulation assays. J Thromb Haemost 2011; 9: 133-9.

50. Baglin T, Hillarp A, Tripodi A, et al. Measuring Oral Direct Inhibitors (ODIs) of thrombin and factor Xa: a recommendation from the Subcommittee on Control of Anticoagulation of the Scientific and Standardisation Committee of the International Society on Thrombosis and Haemostasis. J Thromb Haemost 2013; 11 : 756-60.

51. Czubek U, Góralczyk T, Zalewski J, Undas A. Monitoring of anticoagulant effects of dabigatran in everyday practice: first experience in 32 Polish patients. Pol Arch Med Wewn 2014; 124: 487-9.

52. Zalewski J, Rychlak R, Góralczyk T, Undas A. Rivaroxaban concentration in patients with deep vein thrombosis who reported thrombus progression or minor hemorrhagic complications: first Polish experience. Pol Arch Med Wewn 2014; 124: 553-5.

53. Lindhoff-Last E, Samama MM, Ortel TL, et al. Assays for measuring rivaroxaban: Their suitability and limitations. Ther Drug Monit 2010; 32: 673-9.

54. Undas A, Pasierski T, Windyga J, Crowther M. Practical therapeutic aspects of new oral anticoagulants use in atrial fibrillation. Pol Arch Med Wewn 2014; 124: 124-35.

55. Lippi G, Favaloro EJ. Urgent monitoring of dabigatran plasma levels: sometimes less is more. Pol Arch Med Wewn 2014; 124: 639-40.

56. Pollack CV Jr, Reilly PA, Eikelboom J, et al. Idarucizumab for dabigatran reversal. N Engl J Med 2015; 373: 511-20.

57. Siegal DM, Curnutte JT, Connolly SJ, et al. Andexanet alfa for the reversal of factor Xa inhibitor activity. N Engl J Med 2015; 373: 2413-24.

58. Mazur P, Darocha T, Filip G, et al. Idarucizumab for dabigatran reversal in patients with atrial fibrillation undergoing emergency surgery for acute aortic syndrome. Pol Arch Med Wewn 2016; 126: 579-81.

59. Yeh RW, Sidney S, Chandra $M$, et al. Population trends in the incidence and outcomes of acute myocardial infarction. N Engl J Med 2010; 362: 2155-65.

60. Roffi M, Patrono C, Collet JP, et al. 2015 ESC Guidelines for the management of acute coronary syndromes in patients presenting without persistent ST-segment elevation: Task Force for the Management of Acute Coronary Syndromes in Patients Presenting without Persistent ST-Segment Elevation of the European Society of Cardiology (ESC). Management of Acute Coronary Syndromes in Patients Presenting without Persistent ST-Segment Elevation of the European Society of Cardiology. Eur Heart J 2016; 37: 267-315.

61. Fox KA, Dabbous OH, Goldberg RJ, et al. Prediction of risk of death and myocardial infarction in the six months after presen- 
tation with acute coronary syndrome: prospective multinational observational study (GRACE). BMJ 2006; 333: 1091.

62. Yusuf S, Zhao F, Mehta SR, et al. Effects of clopidogrel in addition to aspirin in patients with acute coronary syndromes without ST-segment elevation. N Engl J Med 2001; 345: 494-502.

63. Fox KA, Carruthers KF, Dunbar DR, et al. Underestimated and under-recognized: the late consequences of acute coronary syndrome (GRACE UK-Belgian Study). Eur Heart J 2010; 31: 2755-64.

64. Wiviott SD, Braunwald E, McCabe $\mathrm{CH}$, et al. Prasugrel versus clopidogrel in patients with acute coronary syndromes. N Engl J Med 2007; 357: 2001-15.

65. Wallentin L, Becker RC, Budaj A, et al. Ticagrelor versus clopidogrel in patients with acute coronary syndromes. N Engl J Med 2009; 361: 1045-57.

66. Oldgren J, Budaj A, Granger CB, et al. RE-DEEM Investigators. Dabigatran vs. placebo in patients with acute coronary syndromes on dual antiplatelet therapy: a randomized, double-blind, phase II trial. Eur Heart J 2011; 32: 2781-9.

67. Alexander JH, Becker RC, Bhatt DL, et al. Apixaban, an oral, direct, selective factor Xa inhibitor, in combination with antiplatelet therapy after acute coronary syndrome: results of the Apixaban for Prevention of Acute Ischemic and Safety Events (APPRAISE) trial. Circulation 2009; 119: 2877-85.

68. Alexander JH, Lopes RD, James S, et al. Apixaban with antiplatelet therapy after acute coronary syndrome. N Engl J Med 2011; 365: 699-708.

69. Mega JL, Braunwald E, Mohanavelu S, et al. ATLAS ACS-TIMI 46 study group. Rivaroxaban versus placebo in patients with acute coronary syndromes (ATLAS ACS-TIMI 46): a randomised, double-blind, phase II trial. Lancet 2009; 374: 29-38.

70. Mega JL, Braunwald E, Wiviott SD, et al. Rivaroxaban in patients with a recent acute coronary syndrome. N Engl J Med 2012; 366 : 9-19.

71. Montagnana M, Danese E, Lippi G. Genetic risk factors of atherothrombosis. Pol Arch Med Wewn 2014; 124: 474-82. 